Check for updates

Cite this: RSC Adv., 2018, 8, 10855

Received 8th January 2018

Accepted 12th March 2018

DOI: 10.1039/c8ra00196k

rsc.li/rsc-advances

\section{Electromagnetic and microwave absorption characteristics of PMMA composites filled with a nanoporous resorcinol formaldehyde based carbon aerogel}

\begin{abstract}
A. Abolghasemi Mahani, ${ }^{a}$ S. Motahari (D) ${ }^{* a}$ and V. Nayyeri (DD ${ }^{b}$
Nanostructured carbons have opened up new perspectives in fields of electromagnetic (EM) applications. The present study aims at the processing of microwave absorbing (MA) materials based on carbon aerogels (CAs) in polymethyl methacrylate (PMMA) matrix to be used in X-band frequency. CAs were synthesized by carbonization of a sol-gel derived organic gel from resorcinol and formaldehyde as starting materials. Microwave attenuation properties of the prepared composites were investigated in terms of CAs particle size distribution (PSD) and mass fraction. To do so, the optimal PSD was initially determined by assessing the EM attenuation performance of the CAs/PMMA composites with constant mass loading (10 wt\%) and differing particle sizes. Next, the EM properties of the selected CAs with the optimal particle size was measured as a function of mass fraction varying from 1 to $15 \mathrm{wt} \%$ in order to obtain a highly efficient CAs based MA. The results indicate that the dielectric loss of CAs composites can be enhanced by optimizing the PSD as well as the mass fraction of CAs. The effective absorption bandwidth of composites containing $10 \mathrm{wt} \%$ of CAs exceeded $3.7 \mathrm{GHz}$ at a very thin thickness of $1.9 \mathrm{~mm}$ indicating that these materials present advantages as microwave absorbers.
\end{abstract}

\section{Introduction}

During the current decade, with the rapid developments in information technology, electromagnetic (EM) wave radiation is drawing global attention as a new source of pollution. ${ }^{1,2}$ To eliminate adverse electromagnetic radiation in healthcare and electronic circuit's performance, much attention has been paid to the development of highly efficient microwave absorber (MA) materials..$^{3-6}$

According to the microwave absorption theory, MA can be divided into dielectric and magnetic loss materials. ${ }^{4}$ In this regard, solid particles such as ferrites, ceramics, and their hybrids are conventional materials widely utilized as MA constituents. However, high density, poor chemical stability, narrow frequency band absorption, and high loading content are the main drawbacks hindering practical applications of the above-mentioned materials. Hence, to overcome such problems, special efforts have been devoted to the development and utilization of new alternatives as lightweight and broadband MA., ${ }^{4,-13}$

Typically, broadband dielectric loss composites containing carbon fillers such as carbon black, carbon fibers, carbon

${ }^{a}$ School of Chemical Engineering, Faculty of Engineering, University of Tehran, P. O. Box: 11365/4563, Tehran, Iran.E-mail: a.m.abolghasemi@ut.ac.ir; smotahari@ut. ac.ir

${ }^{b}$ Antenna and Microwave Research Laboratory, Iran University of Science and Technology, Tehran, Iran.E-mail: nayyeri@iust.ac.ir nanotubes as well as porous carbons exhibits significant performance as MA. This is due to their special features including chemical resistivity, lightweight, and tunable absorbing characteristics. ${ }^{\mathbf{8} 14-17}$ Recently, special efforts have been made to use of porous carbons as efficient MA due to their high specific surface area, porosity, polarizability and multiple scattering properties. Structural properties, chemical functionality as well as particle size distribution (PSD) are some special intrinsic characteristics having the dominant effect on the electromagnetic properties of these types of absorbents., ${ }^{4,8,18-21}$ For example, Huang et al. ${ }^{22}$ investigated the effect of pore texture of sol-gel derived resorcinol-furfural on EM characteristics of the mesoporous carbons. It was concluded that the synthesized porous carbons with a cage-like structure exhibited interesting electromagnetic properties in terms of the real and imaginary parts of the permittivity. Du et al. ${ }^{23}$ examined the carbonization temperature on the electromagnetic absorption properties of the ordered mesoporous carbons in $\mathrm{K}$ band frequency. They found that the pyrolyzed mesoporous carbons at $650^{\circ} \mathrm{C}$ demonstrated a considerable reflection loss of $-27 \mathrm{~dB}$ at $16.1 \mathrm{GHz}$. Li et al. ${ }^{24}$ studied the electromagnetic absorption properties of $\mathrm{CNF}$ and porous-CNF composites. The results confirmed that the composite containing $6 \mathrm{wt} \%$ of porous CNF exhibited the maximum reflection loss of $-31 \mathrm{~dB}$ at $9.7 \mathrm{GHz}$. As yet another example, Yin et al. ${ }^{19}$ reported a successful method in the preparation of mesoporous carbon hollow microspheres 
(PCHMs) with enhancement in electromagnetic absorption. They found that a composite containing 20 wt\% PCHMs demonstrated a competitive electromagnetic absorption performance in the $\mathrm{X}$ band frequency.

Carbon aerogels (CAs) are low-density nano/micro-porous materials, consisting of continuous porosities accompanied by the ordered assembly of carbon particles. Generally, this type of materials produced by carbonization of sol-gel derived organic precursors. $^{25,26}$ They are used in many various engineering applications such as super thermal insulation, hydrogen storage, catalyst applications, and electrochemical double-layer capacitors. High porosity $(>80 \%)$, large surface area $\left(400-2700 \mathrm{~m}^{2} \mathrm{~g}^{-1}\right)$ and tunable structural characteristics are their valuable intrinsic properties. ${ }^{25-28}$ Hence, special efforts have been made to modulate the synthesis and processing conditions to tune the CAs structures for their target applications. Among the large variety of CAs precursors, resorcinol formaldehyde (RF) aerogels are still the primary commercial source to produce CAs. ${ }^{29}$ Concerning the electrical application point of view, a great deal of attention has been paid to investigating and synthesizing high performance 3D interconnected RF-based CAs as targeting materials. ${ }^{30-32}$ However, restricted information exists on the EM wave absorbing properties of RF-based CAs.

The present study aims at producing RF-based CAs as X-band MA components. The samples are synthesized by carbonization of sol-gel derived RF aerogel at $950{ }^{\circ} \mathrm{C}$ under argon (Ar) atmosphere. The influences of PSD and mass fraction are investigated on dielectric and microwave characteristics of the corresponding CAs/PMMA composites. The electromagnetic characterization of CA composites was specified via transmission/reflection line method in the X-band frequency. Initially, the optimal PSD determined by investigating the effective EM attenuation performance, maximum absorption bandwidth below $-10 \mathrm{~dB}$, of the prepared CAs/PMMA composites sharing the same mass loading but different particle sizes. Next, in order to assess the impact of filler content on the MA performance of CAs, the EM attenuation properties of the selected CAs were measured as a function of mass fraction ranging from 1 to $15 \mathrm{wt} \%$. With the best knowledge of the authors, this is the first study about the comprehensive investigation of the effects of PSD and mass fraction on electromagnetic and X-band microwave absorption performance of mesoporous resorcinol formaldehyde based carbon aerogel/PMMA composites.

\section{Experimental}

\section{Materials}

All chemicals including resorcinol ( $99 \%)$, formaldehyde (37\% wt), as starting materials, methyl methacrylate (MMA) and sodium carbonate, as polymer matrix precursor and catalyst, respectively, were purchased from Merck (Merck Chemical Co) and used without extra purification. Deionized water was used as solvent.

\section{Sample preparation}

The organic RF aerogel was prepared by reaction between resorcinol and formaldehyde under basic condition. In a typical procedure, $5 \mathrm{~g}$ resorcinol $(0.0454 \mathrm{~mol})$ was dissolved in $16.882 \mathrm{~g}$ (0.93 mol) of distilled water. After mixing and homogenizing with $9.74 \mathrm{~g}$ ( $0.68 \mathrm{~mol})$ of $37 \mathrm{wt} \%$ aqueous formaldehyde, the prepared solution was mixed with $0.954 \mathrm{~g}$ of sodium carbonate solution with the molarity of 0.0119 . The final solution was stirred for $30 \mathrm{~min}$ and poured into glass vials which were then sealed and cured at $60{ }^{\circ} \mathrm{C}$ for 2 days for the purpose of both gelation and the aging of the wet gels. Over the period of 2 days, the initially colorless solution became dark red when the RF wet gel formed. To dry and control the exerted capillary pressure, the samples with $180 \mathrm{ml}$ of water were placed in a stainless steel vessel. The insulated system was heated gradually to reach the elevated temperature and pressure of $200{ }^{\circ} \mathrm{C}$ and $16 \mathrm{bar}$, respectively, followed by a sudden drop in system pressure. Under such condition, the vessel pressure reached the atmospheric pressure at constant temperature $\left(200^{\circ} \mathrm{C}\right)$. Through this operation, RF aerogels with lower degree of shrinkage can be produced.

The carbonization process of the RF aerogel was conducted as follows: the samples were placed in an electrical ceramic type furnace and heated from ambient temperature to $600{ }^{\circ} \mathrm{C}$ under Ar atmosphere at a heating rate of $7^{\circ} \mathrm{C} \mathrm{min}^{-1}$. Then the samples were heated to $950{ }^{\circ} \mathrm{C}$ at a heating rate of $3{ }^{\circ} \mathrm{C} \min ^{-1}$ with no delay. Finally, the samples were held at $950{ }^{\circ} \mathrm{C}$ for $2 \mathrm{~h}$ and then the furnace was cooled down to room temperature naturally under the inert condition. Finally, the synthesized CAs were ground into the fine particles using a planetary ball mill. The PSD of the samples was controlled by variation of milling time from 1 to $6 \mathrm{~h}$ and the samples were named as Gr- $n$, in which $n$ represents the grinding time in $\mathrm{h}$. For the purpose of dielectric characterization, the prepared CAs was dispersed in a MMA monomer with the help of an ultrasonic bath and a magnetic stirrer. Then, CAs/MMA monomer mixtures were casted into a rectangular shaped mold with a certain size fitting the waveguide dimensions (i.e. $0.4 \times 0.9$ inch $^{2}$ ) and cured in an oven for $12 \mathrm{~h}$. The optimal PSD was determined by investigating the EM attenuation performance of the prepared CAs/PMMA composites in term of maximum effective absorption bandwidth. After that, CAS with the optimal PSD was selected for further experiments in order to specify the most effective weight percent. At this stage, composites with different load of CAs from 1 to $15 \mathrm{wt} \%$ were prepared and examined.

\section{Characterization methods}

The characterization of the as-prepared CA powders was specified by implementing various techniques. The graphitization level of the prepared CAs was investigated by X-ray diffraction method (X'Pert MPD, Philips, Holland, using $\mathrm{Cu}-\mathrm{K} \alpha$ radiation). Chemical bond formation in prepared samples were studied by FTIR spectroscopy under transmittance mode, in the range of 400-4000 $\mathrm{cm}^{-1}$ using (Bruker Equinox 55LS 101 series, Germany). Morphology study of the CAs samples was conducted using FE-SEM (TESCAN, MIRA3 LMH/LMU). The PSD of CAs was evaluated by Fritsch particle size analyzer (MERC, Germany). The measurement of specific surface areas and pore analysis of CAs were performed at $77 \mathrm{~K}$ by nitrogen $\left(\mathrm{N}_{2}\right)$ 
physisorption on a BELSORP-mini II (Bell Japan Inc.) analyzer. The microwave absorbing performances of CA composites were evaluated on an Agilent E8362B vector network analyzer in the $\mathrm{X}$-band frequency region. The porosity percentage of the prepared CAs was measured using the following formula ${ }^{33,34}$ and found to be $82 \%$.

$$
\text { Porosity }(\%)=\left[1-\left(\frac{\rho_{\mathrm{a}}}{\rho_{\mathrm{s}}}\right)\right] \times 100
$$

where $\rho_{\mathrm{a}}$ and $\rho_{\mathrm{s}}$ are apparent (bulk) and skeletal density of the aerogels, respectively. The skeletal density was measured by helium pycnometer (HelPyc Che-1701, Omid Petro Energy Khavaran CO) and found to be $1.831\left(\mathrm{~g} \mathrm{~cm}^{-3}\right)$. The apparent densities of the CAs were evaluated by calculating the ratio of the measured mass to unit volume of the carbons.

\section{Electromagnetic considerations}

In this research, the electromagnetic and reflection loss properties of CAs composites are calculated according to transmission line theory and the perfect electric conductor (PEC) model, respectively. As implied by the model, wave impedance at the interface of air/absorber is expressed by the following equation; $;, 35,36$

$$
Z_{\text {in }}=Z_{0} \sqrt{\frac{\mu_{\mathrm{r}}}{\varepsilon_{\mathrm{r}}}} \times \tanh \left[\left(\frac{-\mathrm{j} 2 \pi}{C}\right) \times \sqrt{\frac{\mu_{\mathrm{r}}}{\varepsilon_{\mathrm{r}}}} \times f \times d\right]
$$

where $\mu_{\mathrm{r}}$ and $\varepsilon_{\mathrm{r}}$ represent complex relative magnetic permeability and electrical permittivity of the absorber, respectively. $Z_{\text {in }}, Z_{0}, f, C$ and $d$ stand for the wave impedance at interface, wave impedance in free space $(377 \Omega)$, the wave frequency, velocity of the light and the thickness of the absorber, respectively. The reflection loss (RL) of the absorber can be calculated using the following equation: $:^{4,22,37}$

$$
\mathrm{RL}(\mathrm{dB})=-20 \log \left|\frac{Z_{\text {in }}-Z_{0}}{Z_{\text {in }}+Z_{0}}\right|
$$

The dip in RL indicates higher absorption ability of EM wave at a certain frequency for a particular sample thickness.

It is well known that, the thickness of EM absorbers is a determining factor to both the position and intensity of the maximum RL. ${ }^{4}$ Hence, in this research the thickness of samples is optimized based on geometrical effect for simplicity of comparison. Under geometrical effect, microwave absorbers show their maximum absorption loss, which is ascribed to the thickness of absorber $\left(t_{\mathrm{m}}\right)$ when the following equation is satisfied; ;,24,38

$$
t_{\mathrm{m}}=n \frac{\lambda_{m}}{4} \quad\left(n=1,3,5, \ldots \text { and } \quad \lambda_{\mathrm{m}}=\frac{\lambda_{0}}{\sqrt{\left|\varepsilon_{\mathrm{r}} \mu_{\mathrm{r}}\right|}}\right)
$$

where $\lambda_{\mathrm{m}}$ and $\lambda_{0}$ stand for the wave length in the absorber and in free space at a certain frequency, respectively. $\left|\varepsilon_{\mathrm{r}}\right|$ and $\left|\mu_{\mathrm{r}}\right|$ are also moduli of complex permittivity and permeability, respectively. In this research, with respect to the geometrical effect, the thickness of the absorbers is fixed in a way that the maximum RL of EM occurs at $10 \mathrm{GHz}$.

\section{Results and discussions}

\section{Pore texture analysis}

The nitrogen adsorption-desorption isotherms as well as pore size distributions for the grinded CAs samples are shown in Fig. 1. As illustrated, the sorption isotherm shows a rapid rise at low relative pressures, which then gets horizontal plateau at higher relative pressures. According to IUPAC classification this behavior is characteristic of type I isotherm, which confirms that during carbonization, micropores were developed in the CAs. ${ }^{39,40}$

Adsorption-desorption isotherms of the CAs also exhibits a type-IV IUPAC with hysteresis loop of type-H3. This type of hysteresis loop with the out-of-level $\mathrm{N}_{2}$ adsorption at high relative pressures $\left(P / P_{0}=0.99\right)$ is characteristic of capillary condensation in mesoporous structures, as well as the plate-like particles which give rise to slit-shaped pores. ${ }^{39,41,42}$ This finding is further proved by estimated pore size distribution from $\mathrm{BJH}$ method.

Pore size distribution of CAs is evaluated by BJH method, which is present in the inset of Fig. 1. As depicted, in all samples a broad pore size distribution from micro to mesopore size is observed, which is varying from 1 to $40 \mathrm{~nm}$ and not distributed uniformly. Main pore sizes in CAs were found to be 1 and $40 \mathrm{~nm}$, which is indicative of micro and mesopore size, respectively. Similarity of the pore size distribution for the samples grinded in different time is an evidence confirming that grinding time does not affect the pore texture of CAs.

The variation of specific surface areas of the samples as a function of grinding time is tabulated in Table 1. Although increasing of grinding time creates smaller grains, it does not affect total specific surface area significantly. Specific surface area of CAs increased about $10 \%$ from 561 to $618\left(\mathrm{~m}^{2} \mathrm{~g}^{-1}\right)$ when the grinding time varied from 1 to $6 \mathrm{~h}$, respectively.

\section{Morphology study and particle size analysis}

Effect of grinding time on morphology and PSD of the prepared CAs is illustrated in FE-SEM graph (Fig. 2). The FE-SEM

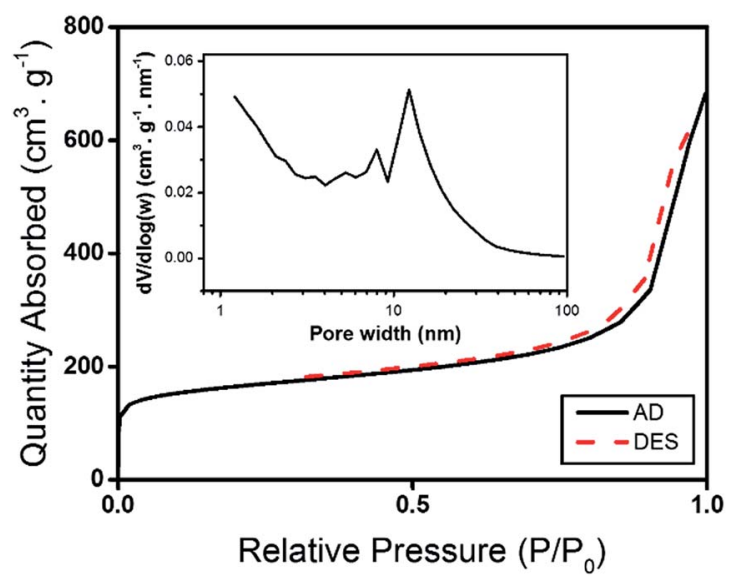

Fig. 1 Effect of grinding on textural properties of CAs (nitrogen adsorption-desorption isotherms as well as pore size distribution analysis). 
Table 1 Effect of grinding on the specific surface area of CAs

\begin{tabular}{ll}
\hline Sample & Surface area $\left(\mathrm{m}^{2} \mathrm{~g}^{-1}\right)$ \\
\hline RF aerogel & 370 \\
Gr-1 & 561 \\
Gr-2 & 587 \\
Gr-4 & 610 \\
Gr-6 & 618
\end{tabular}

micrographs confirmed that the porous CAs are made of interconnected nano-metric particles. The average particle size is calculated using analytical method and found to be $\sim 43.5 \mathrm{~nm}$ under the spherical shape assumption of carbon particles. The observed pore size values were also consistent with pore size distribution of $\mathrm{N}_{2}$ physisorption measurements.

The structural observation revealed that all grinded CAs have highly ordered 3D porous structure with the same values of pores and particle sizes. On the other hand, as indicated in BET and $\mathrm{BJH}$ analysis, morphology observation revealed that, pore characteristic of CAs was not affected by grinding process.

The PSD analysis from CAs samples also confirms what the SEM graphs show. PSD of the samples decreases while the grinding time increases. As illustrated in Fig. 2 the amount of the particles with PSD below $10 \mu \mathrm{m}$ are $45 \%, 63 \%, 74 \%$ and $82 \%$ in the samples grinded for 1, 2, 4 and $6 \mathrm{~h}$, respectively.

The observed pore size values were also consistent with pore size distribution of $\mathrm{N}_{2}$ physisorption measurements. The structural observation revealed that all grinded CAs have highly ordered 3D porous structure with the same values of pores and particle sizes. On the other hand, as indicated in BET and BJH analysis, morphology observation revealed that, pore characteristic of CAs was not affected by grinding process. The PSD analysis from CAs samples also confirms what the SEM graphs show. PSD of the samples decreases while the grinding time increases. As illustrated in Fig. 2 the amount of the particles with PSD below $10 \mu \mathrm{m}$ are $45 \%, 63 \%, 74 \%$ and $82 \%$ in the samples grinded for 1, 2, 4 and $6 \mathrm{~h}$, respectively.

\section{XRD Analysis}

In order to study the development of crystallinity in CAs, the $\mathrm{XRD}$ analysis was carried out and the results is presented in Fig. 3. Since all XRD patterns of CAs are similar to each other, here only one of them is presented as an example. The two broad peaks positioned at $2 \theta=24^{\circ}$ and $42^{\circ}$ correspond to the
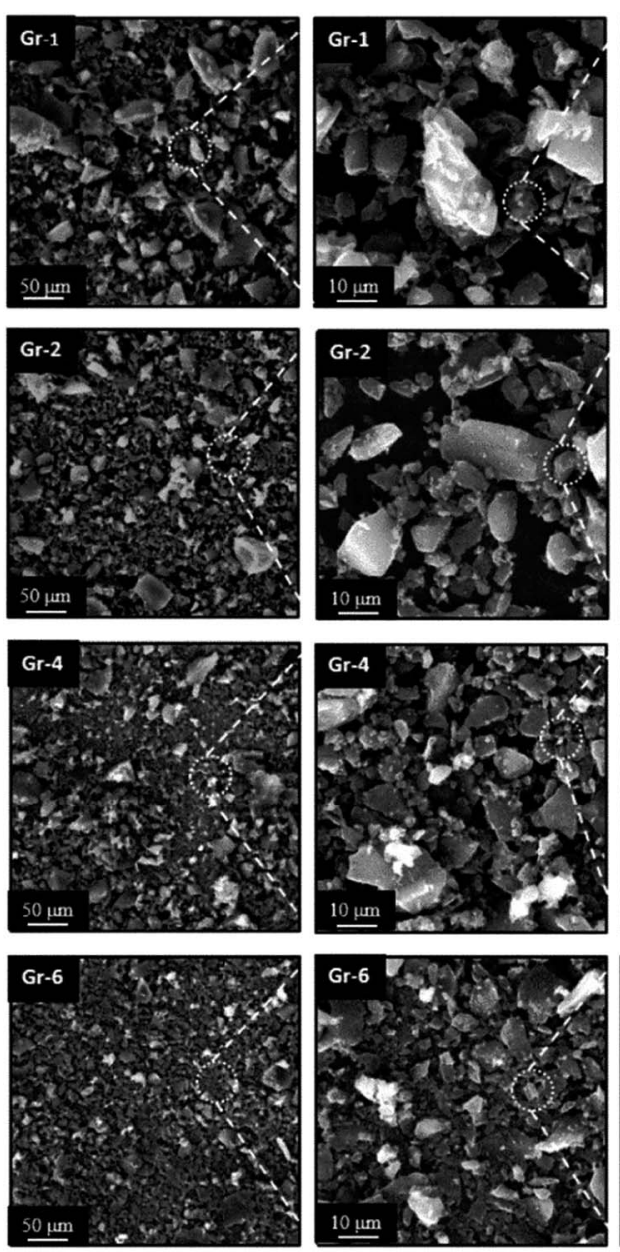
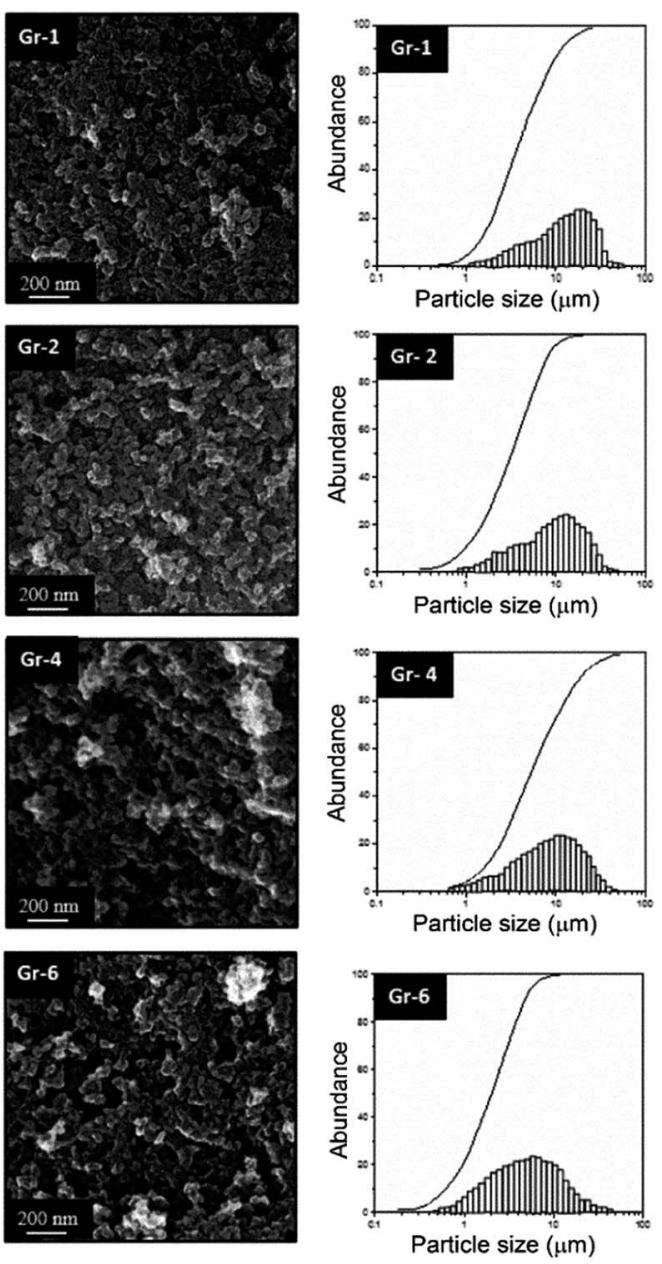

Fig. 2 Effect of milling time on the morphology and PSD of CAs. 


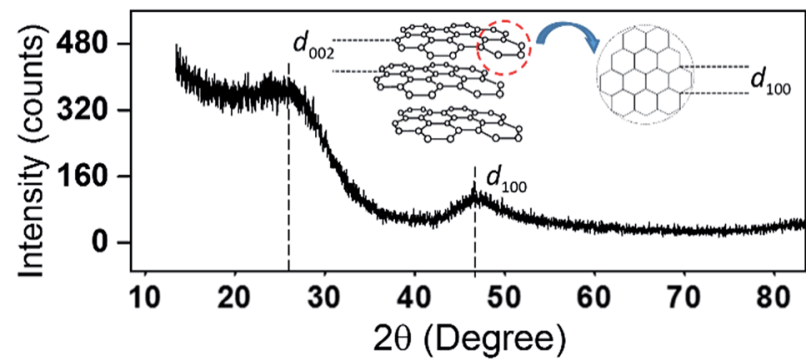

Fig. 3 XRD spectra of the synthesized CAs.

reflections of (002) and (100) planes of graphite, respectively. ${ }^{22,29,35}$ Since the peak intensity of $d_{002}$ plane in CAs is weak, it is concluded that the CAs structure consists of low degree of crystallinity.

\section{FTIR analysis}

FTIR spectroscopy is used to study surface chemistry and the changes in chemical functional groups during the pyrolysis of RF aerogel. The FTIR spectra of RF aerogel and corresponding CAs are shown in Fig. 4a and Fig. 4b, respectively. Since all FTIR spectra of the grinded CAs are similar to each other, here only one of them is presented. The broad peak at $3422 \mathrm{~cm}^{-1}$ corresponds to the $-\mathrm{OH}$ group on the resorcinol ring in $\mathrm{RF}$ aerogel. The peaks at 1460 and $2925 \mathrm{~cm}^{-1}$ are related to the stretching vibration of $\mathrm{CH}_{2}$ groups. The peak at $1614 \mathrm{~cm}^{-1}$ also corresponds to the benzene ring stretching. The observed peaks at 1091 and $1220 \mathrm{~cm}^{-1}$ confirm the stretching of C-O-C linkage between resorcinol molecules which is created during sol-gel reaction. ${ }^{43-45}$ As shown in Fig. 4b pyrolysis process has a crucial effect on the chemical nature of the original RF aerogel.

For example, the hydroxyl peak characteristic at $3422 \mathrm{~cm}^{-1}$ becomes more intensive and a new peak can be observed at $3553 \mathrm{~cm}^{-1}$.

These significant changes in hydroxyl group intensity and a new peak at $3553 \mathrm{~cm}^{-1}$ may originated from the thermal decomposition of $\mathrm{C}-\mathrm{O}-\mathrm{C}$ linkage and formation of $=\mathrm{CH}_{2}$ and $\mathrm{CH}_{2} \mathrm{OH}$ chemical bonds, respectively. This is further supported

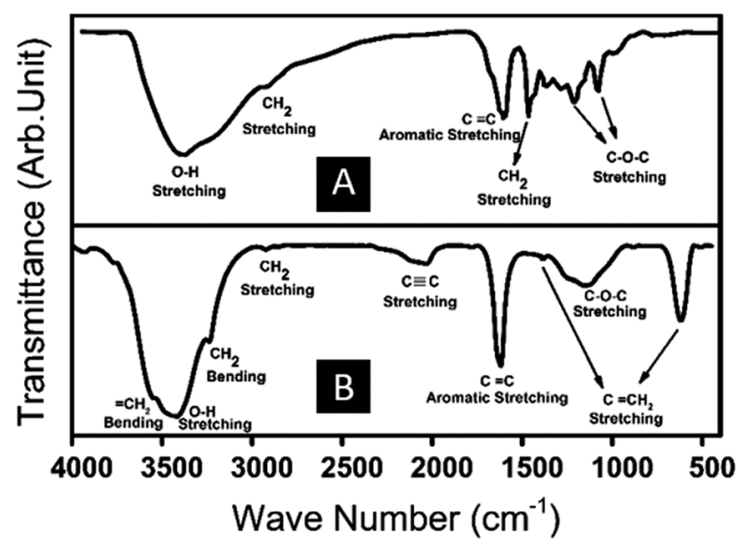

Fig. 4 FTIR spectra of RF aerogel (A) and corresponding CAs (B). by the observed new intense peak in CAs at 660 and $1383 \mathrm{~cm}^{-1}$ due to the presence of $\mathrm{C}=\mathrm{CH}_{2}$ groups that are not visible in FTIR spectra of RF aerogel. As shown in Fig. 4b, weak and broad peak in the wave number region from 1098-1367 is characteristic of $\mathrm{C}-\mathrm{O}-\mathrm{C}$ linkage, implying that some linkages are intact during pyrolysis. The observed weak peak at $3238 \mathrm{~cm}^{-1}$ is indicative of bending vibration of $\mathrm{C}-\mathrm{H}$. The new observed peak at $2198 \mathrm{~cm}^{-1}$ can be attributed to $-\mathrm{C} \equiv \mathrm{C}$ - bonds which means that some benzene rings are broken under the heating process and new $\mathrm{C} \equiv \mathrm{C}$ bonds are formed at the broken ends. Finally, presence of an intensive aromatic peak at $1614 \mathrm{~cm}^{-1}$ implies that the chemical structure of the prepared CAs are uniformly composed of benzene ring arrangement. ${ }^{43-47}$

\section{Electromagnetic properties}

Dielectric constant. Complex permittivity $\left(\varepsilon_{\mathrm{r}}\right)$ is the most important characteristic to investigate the intrinsic reasons for microwave absorption performance of dielectrics, which is expressed as $\varepsilon_{\mathrm{r}}=\varepsilon_{\mathrm{r}}^{\prime}-\mathrm{j} \varepsilon^{\prime \prime}{ }_{\mathrm{r}}$. According to the free electron theory, $\varepsilon^{\prime}$ r presents storage capability of the materials while $\varepsilon^{\prime \prime}{ }_{\mathrm{r}}$ reflects the attenuation characteristic at a certain frequency of EM wave. ${ }^{8,36,48}$

Fig. 5 illustrates the variation of dielectric constants of the CAs/PMMA composites containing $10 \mathrm{wt} \%$ of CAs as a function of frequency and PSD. As can be inferred from Fig. 5, from Gr-1 to Gr-6 samples both imaginary and real parts of permittivity show a relative escalation when the PSD of CAs turns into the finer particles. When the grinding time duration increased from
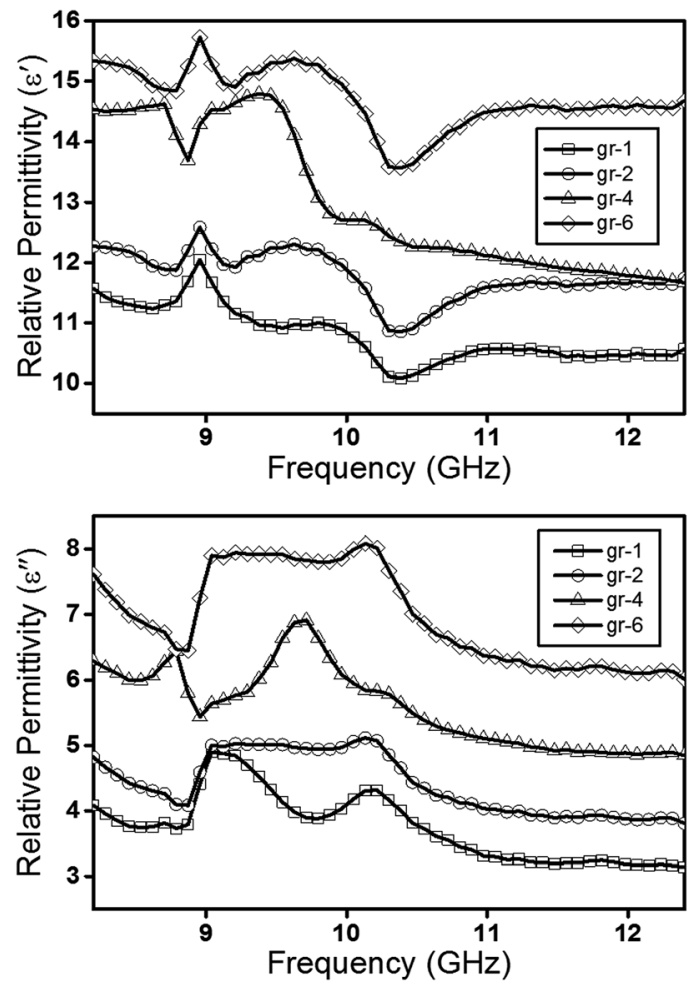

Fig. 5 Variation of dielectric constants of the CAs/PMMA composites containing $10 \mathrm{wt} \%$ of CAs as a function of frequency and PSD. 
1 to $6 \mathrm{~h}$, the $\varepsilon^{\prime}$ r values shown a relative $26 \%$ rise from 14.4 to 18.16 at $10 \mathrm{GHz}$. As indicated in Fig. 5, by only enhancing the time duration from 4 to $6 \mathrm{~h}$ less variation could be recorded for $\varepsilon^{\prime}{ }_{\mathrm{r}}$ (almost $\left.10 \%\right)$. By increasing the grinding time $\varepsilon^{\prime \prime}{ }_{\mathrm{r}}$ also has led to similar alteration as those happening in real part of permittivity. The reason behind such variation in permittivity parameters is closely related to the theoretical density of composite powders and the rule of mixtures. According to theoretical density, when CAs are milled into fine particles the density of the proceeded CAs decreases. ${ }^{7,19}$ Hence, in composites with the same mass content of CAs, the volume fraction of the prepared CAs with the longer milling time is higher than those proceeded carbons under the shorter milling time. This would lead to a better conductivity in the samples, which in turn enhances the permittivity level.

It should be noted that, in this research the CAs are dielectric absorbers with no magnetic susceptibility so the values of complex magnetic permeability can be disregarded (i.e. $\mu_{\mathrm{r}}^{\prime}=1$, $\left.\mu^{\prime \prime}{ }_{\mathrm{r}} \approx 0\right)$.

In dielectric absorbers, the dielectric loss tangent $\left(\tan \delta_{\varepsilon}=\right.$ $\left.\varepsilon^{\prime \prime} / \varepsilon^{\prime}\right)$ is an important parameter to evaluate the EM attenuation capability of absorber when the permittivity meets the impedance matching requirements. ${ }^{49}$ The $\tan \delta_{\varepsilon}$ of CAs composites were calculated based on the real and imaginary parts of permittivity and shown in Fig. 6 . The tan $\delta_{\varepsilon}$ of samples exhibited nonlinear behavior with bimodal characteristic. The positions where $\tan \delta_{\varepsilon}$ fluctuations occur are exactly similar to the resonance peaks of $\varepsilon^{\prime \prime}{ }_{\mathrm{r}}$, which is attributed to interfacial polarization. ${ }^{50}$ The values of $\tan \delta_{\varepsilon}$ increased gradually from 0.36 to 0.55 , at $10 \mathrm{GHz}$, as the milling time increased from 1 to $6 \mathrm{~h}$.

EM absorbing characteristic. Microwave attenuation is a process of depletion and transformation of electromagnetic power into thermal energy. ${ }^{51}$ Generally, the measured dielectric parameters at the desired frequency are used to determine the RL of incident EM wave in materials. ${ }^{4,17,20,49}$ Fig. 7 shows the calculated RL of prepared CAs composites in the range of 8.212.4 GHz as a function of PSD. It can be clearly seen that the milling time has noticeable effects on the microwave absorbing performance of CAs composites. It was observed that except Gr6 samples, effective absorption bandwidth become wider when the PSD of CAs turns into finer particles. The results also show that, the minimum reflection loss typically declined when the

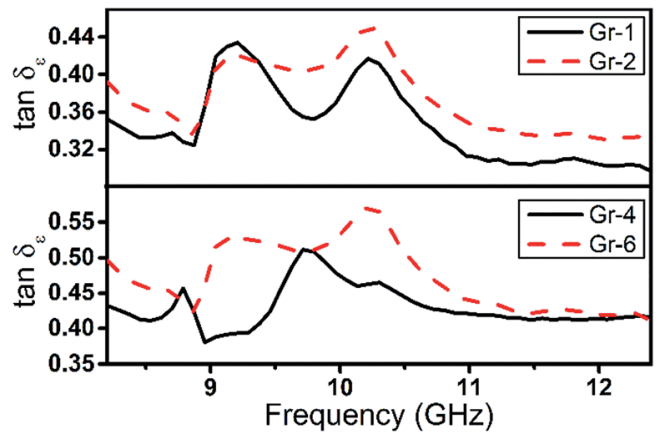

Fig. 6 Loss tangent $\left(\tan \delta_{\varepsilon}\right.$ ) of the prepared CAs as a function of frequency and milling time.

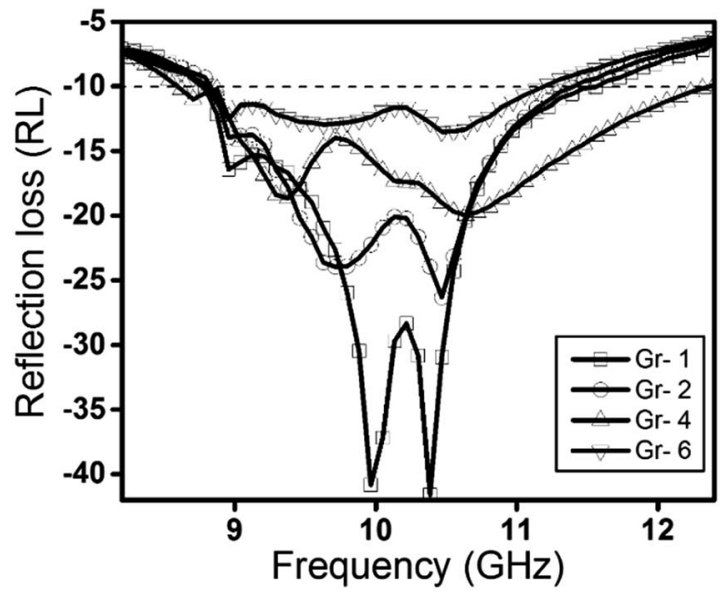

Fig. 7 Minimum RL of the composites containing 10 wt\% CAs as a function of frequency and milling time.

time of milling increases. On the other hand, when the prepared CAs have the larger particle sizes (see SEM analysis), their corresponding composites exhibit stronger RL. The reason for such observation might be hidden in the effective permittivity of medium. According to the Maxwell-Garnett (MG) theory, the value for effective permittivity in a medium can be measured from the following formula; ${ }^{52-55}$

$$
\varepsilon_{\mathrm{eff}}^{\mathrm{MG}}=\varepsilon_{1} \frac{\left(\varepsilon_{2}+2 \varepsilon_{1}\right)+2 p\left(\varepsilon_{2}-\varepsilon_{1}\right)}{\left(\varepsilon_{2}+2 \varepsilon_{1}\right)-p\left(\varepsilon_{2}-\varepsilon_{1}\right)}
$$

where, $\varepsilon_{1}, \varepsilon_{2}$ stand for the permittivity of materials at solid and gas states, respectively and $p$ is the volume fraction of the guest in the effective medium. Here, one can realize that the permittivity of the gas is considered as 1 , while solid state materials have permittivity values higher than 1 (ref. 53). Thus, from the MG theory it can be concluded that in a medium as MA, the effective permittivity would decrease with increasing the void content. In the current study, from the BET and SEM analysis we find that the prepared CAs have porous characteristic with the broad pore size distribution from micro to meso size regions. Thus, in such porous structure, the effective permittivity of CAs composites declines, which would improve impedance matching of CAs composites and enhance EM wave absorption especially when the particle sizes are larger. On the contrary, the milling process has a destructive effect on the porous structure of CAs when they turn into smaller particle sizes. Therefore, both electrical conductivity and effective permittivity increase, hindering the entrance possibility of EM waves inside the CAs composites. ${ }^{\mathbf{3 , 2 4}}$ Moreover, in addition to general absorption mechanisms, multiple reflections of microwaves in CAs composites also lead to an additional loss mechanism of EM energy, which is based on the interference of reflected waves inside CAs particles. ${ }^{\mathbf{8 , 1 8 , 2 2 , 4 8 , 5 2}}$ In comparison with the typical carbon-based absorbers, depends on synthesis procedure CAs possess pores with different sizes and shapes within their structure, which could provide additional pathway for the absorption of EM waves. ${ }^{22}$ Hence, under the above circumstances, preparation of CAs composites with larger 
particle sizes results in better impedance matching condition under which the EM waves are retract and attenuate when penetrate through the carbon particles. It can be concluded from the results that at constant mass loading of $10 \mathrm{wt} \%$ the $\mathrm{Gr}-$ 1 sample displays the strongest microwave absorbing capability of $\sim-42 \mathrm{~dB}$ at $10 \mathrm{GHz}$ while $\mathrm{Gr}-4$ sample shows the widest effective absorption bandwidth of $3.7 \mathrm{GHz}$ (from 8.5 to $11.5 \mathrm{GHz}$ ) with RL less than $-10 \mathrm{~dB}$.

To investigate the effect of filler content on MA performance of the prepared composites, the dielectric and electromagnetic and attenuation characteristics of CAs/PMMA composites were measured as function of CAs mass loading. For this purpose, desire amounts of $\mathrm{Gr}-4$ samples, as CAs with the widest effective absorption wide band, were mixed with PMMA monomer to prepare composites with varied mass fractions of CAs from 1 to 15 wt $\%$. As shown in Fig. 8, both $\varepsilon^{\prime}$ and $\varepsilon^{\prime \prime}{ }_{\mathrm{r}}$ of the CAs composites increased gradually and reached a maximum as the mass loading of CAs increased from $1 \mathrm{wt} \%$ to $15 \mathrm{wt} \%$. It is worth mentioning that by increasing CAs mass fraction solely from 5 to $7 \mathrm{wt} \%$, the values for $\varepsilon_{\mathrm{r}}^{\prime}$ and $\varepsilon^{\prime \prime}{ }_{\mathrm{r}}$ parameters exhibited a sudden rise from 8 and 1.5 (at $10 \mathrm{GHz}$ ) to 11.2 and 4.3, respectively. Meanwhile, further increase in CAs mass fraction lead to less significant enhancement of the aforementioned parameter values. The sudden rise in the values of $\varepsilon^{\prime}$ and $\varepsilon^{\prime \prime}{ }_{\mathrm{r}}$ might be due to reaching the electrical percolation point in CAs
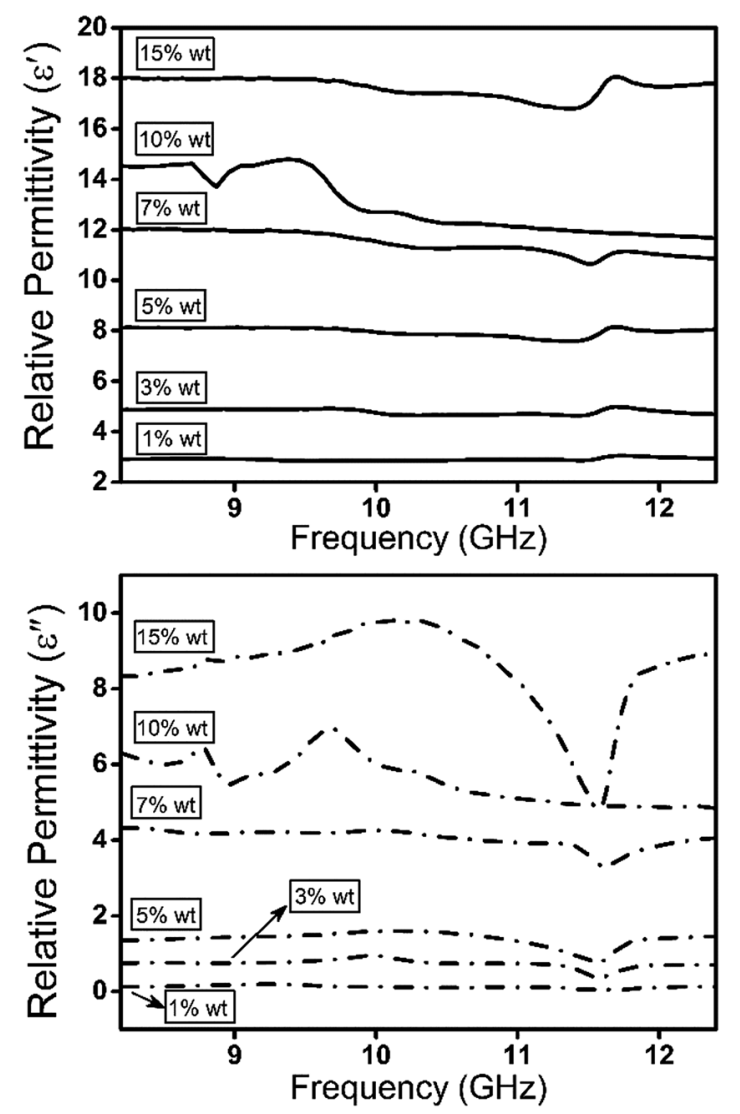

Fig. 8 Variation of dielectric constants of the CAs/PMMA composites as a function of frequency and CAs mass loading. composites along with the formation of a 3D conductivity network in PMMA matrix. ${ }^{56-58}$ Beside other parameters influencing attenuation property of EM waves, reaching electrical percolation can significantly increase conduction loss in composites filled with CAs particles and in turn promote the $\varepsilon^{\prime \prime}{ }_{\mathrm{r}}$ value significantly ( 190\%).

Fig. 9, illustrates the RL of the prepared composites with different CAs content. As shown in Fig. 9, when the composites are combined with the low content of CAs, the corresponding composites show weak RL. On the contrary, by increase in CAs content, RL values show the enhanced attenuation performance especially when the CAs content reach the mass fraction level of $7 \mathrm{wt} \%$ or higher, which is corresponding to the electrical percolation point. As revealed in Fig. 9, maximum RL of composites improved from -0.6 to $-48 \mathrm{~dB}$ when the CAs mass fraction varied from 1 to $7 \mathrm{wt} \%$ and declined to $-10.8 \mathrm{~dB}$ (at $10 \mathrm{GHz}$ ) when CAs mass loading varied from 7 to $15 \mathrm{wt} \%$. As mentioned previously, materials with a higher value of $\varepsilon^{\prime \prime}{ }_{\mathrm{r}}$ suggest enhanced overall EM losses, which induce higher attenuation characteristic. However, as presented in Fig. 9, relative to the composite containing $7 \mathrm{wt} \%$ of CAS, prepared composites under the excessive mass fraction of CAs from $7 \mathrm{wt} \%$ possess weaker absorbing capability.

On the other hand, when the mass fraction of CAs increases from 7 to $15 \mathrm{wt} \%$ the $\mathrm{RL}$ of the CAs composites decreases from -48 to $-10.8 \mathrm{~dB}$ (at $10 \mathrm{GHz}$ ). To explain this behavior, impedance matching of the composites is another key factor to estimate the EM absorption performance, which should be balanced between the medium of propagation and absorber surface. The impedance matching level of CAs composites containing different content of CAs can be calculated in the following equation and is presented in Fig. 10;59-61

$$
\Delta=\left|\sinh ^{2}(K f d)-M\right|
$$

where $K$ and $M$ values could be determined from complex dielectric parameters. Smaller delta value implies better EM impedance matching. ${ }^{59}$

As revealed in Fig. 10, when CAs contents increased from 1 to $7 \mathrm{wt} \%$ the $\Delta$ value decreased from 3.25 to the minimum value of

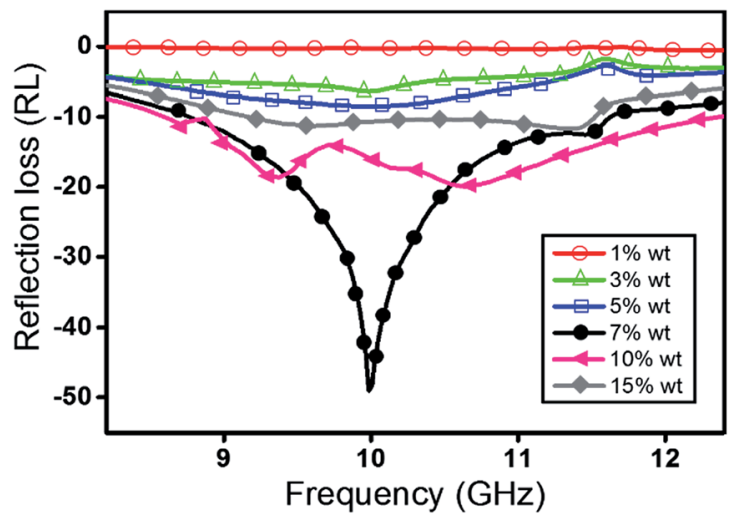

Fig. 9 Variation of minimum reflection loss for the prepared CAs/ PMMA composites as a function of frequency and CAs mass loading. 


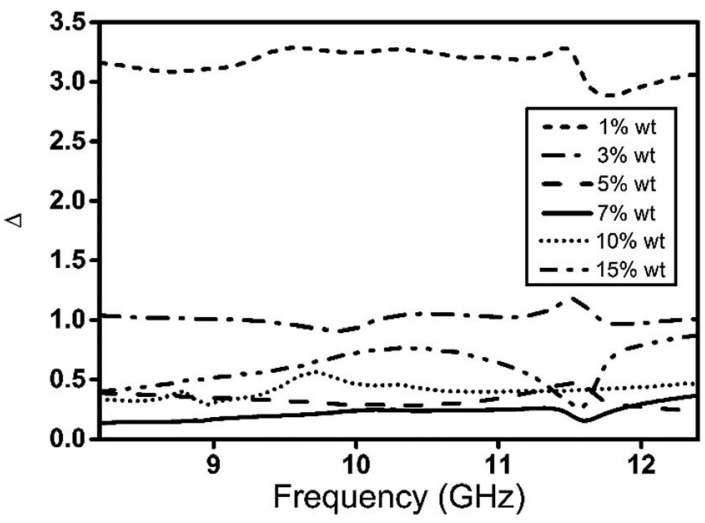

Fig. 10 Variation of $\Delta$ value for the prepared CAs/PMMA composites as a function of frequency and CAs mass loading.

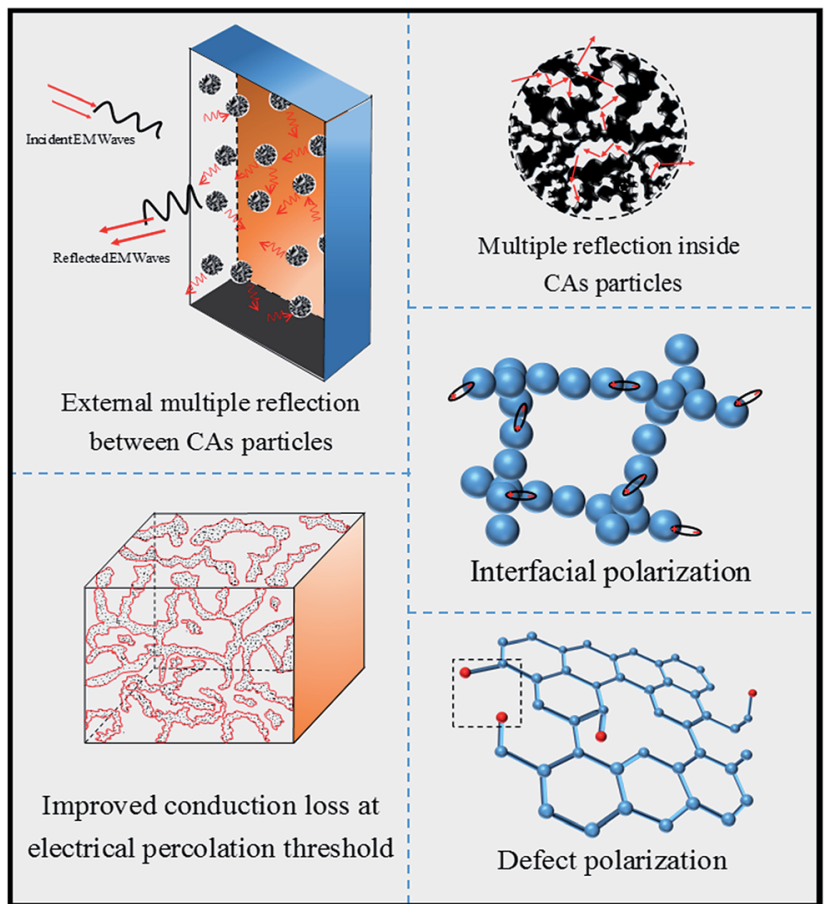

Fig. 11 Mechanisms of EM attenuation in CAs/PMMA composites.
0.22 (at $10 \mathrm{GHz}$ ) implying better impedance matching in CAs/ PMMA composites. On the contrary, the value of $\Delta$ for composites containing 10 and $15 \mathrm{wt} \%$ of CAs increased again to a maximum of 0.72 , which implies poor impedance matching. Under such conditions, a strong reflection of EM wave occurs when the EM waves meet the surface of the absorber, which would restrict the absorption of EM waves inside the absorber.

It should be noted that impedance mismatching is also another reason for decreasing the RL of CAs composites, when the CAs particles proceeded under longer milling time (see Fig. 7).

To better understanding the reason for high attenuation performance in CAs composites, Fig. 11, illustrates the possible absorption mechanisms of EM waves in the prepared samples. Firstly, when the EM waves retract into the CAs composites, the external multiple reflections accompanied with the conduction loss are the primary attenuation mechanisms of the prepared composites. Formation of a conductive network at percolation threshold improves MA performance of CAs composites by migration and hopping the electrons through the composites. Secondly, presence of pores in CAs can cause multiple reflection and scattering of EM waves inside the CAs particles which would in turn prolong the travelling path of EM waves and transforming more EM energy into heat. Besides multiple scattering and conduction loss mechanisms, polarization is also another important factor for excellent MA ability of CAs composites. In case of CAs, interfacial polarization occurs due to the existence of multiple interfaces and the difference in electrical conductivity between carbon particles-PMMA, carbon-carbon clusters, and the carbon particles-trapped air in the pores. Moreover, nanoporous structure of CAs with high specific surface area could dramatically increase the probability of polarization. High specific surface area of CAs causes the formation of more defects in the pore walls and thus brings more interface polarization.

Table 2, reflects a comparison between the prepared CAs composites and the recently reported porous carbon-based composites. It is well known that, effective absorption gap accompanied with RL are the most important parameters for microwave absorbers. The excellent MA performance of CAs composites is closely related to the well-tuned dielectric characteristics as well as the synergetic effect of CAs morphology. Formation of CAs with such porous characteristic accompanied

Table 2 Comparison of MA effectiveness of some porous carbon based composites

\begin{tabular}{|c|c|c|c|c|c|c|}
\hline Type of filler & Filler loading & $\begin{array}{l}\text { Optimal } \\
\text { thickness }[\mathrm{mm}]\end{array}$ & $\begin{array}{l}\text { Frequency } \\
\text { band }\end{array}$ & $\begin{array}{l}\text { Maximum RL } \\
{[\mathrm{dB}]}\end{array}$ & $\begin{array}{l}\text { Absorption } \\
\text { bandwidth [GHz] }\end{array}$ & Ref. \\
\hline CAs & $7 \mathrm{wt} \%$ & 2.2 & $\mathrm{X}$ & $\sim 48$ & 3.7 & This study \\
\hline $\begin{array}{l}\text { Walnut shell-derived nano-porous } \\
\text { carbon }\end{array}$ & $70 \mathrm{wt} \%$ & 2 & $\mathrm{X}$ & 42.5 & 1.76 & 52 \\
\hline 3D MWCNT/graphene hybrid & $2 \mathrm{wt} \%$ & 2 & $\mathrm{X}$ & 26 & 1.6 & 50 \\
\hline Carbon fiber/nano- $\mathrm{Fe}_{2} \mathrm{O}_{3}$ 3D structure & $25 \mathrm{wt} \%$ & 2 & $\mathrm{X}$ & 11 & 2 & 63 \\
\hline
\end{tabular}


with formation of conductive network enhanced the MA properties of CAs composites in terms of absorption intensity and effective bandwidth.

\section{Conclusion}

In this series of experiments, dielectric and MA characteristics of sol-gel derived RF based CAs/PMMA composites was investigated as a function of PSD and CAs mass loading. The provided data suggest that both PSD and filler content play dominant role in tuning EM absorption performance of CAs composites. The prepared CAs composites exhibit superior performance at a lower mass loading, thickness and broadband absorption characteristic. Combination of the above properties indicates a promising perspective for the implementation of CAs composites to development of lightweight high performance MA. The impedance matching condition and RL of the composites were improved by tuning the addition amount of CAs due to the enhancement of interfacial polarization, dipolar polarization and formation of conductive network. It can be concluded from the results that at constant mass loading of $10 \mathrm{wt} \%$ the $\mathrm{Gr}-1$ sample displays the strongest microwave absorbing capability of $\sim-42 \mathrm{~dB}$ at $10 \mathrm{GHz}$ while Gr-4 sample shows the widest effective absorption bandwidth of $3.7 \mathrm{GHz}$ (from 8.5 to $11.5 \mathrm{GHz}$ ) with RL less than $-10 \mathrm{~dB}$.

\section{Conflicts of interest}

There are no conflicts to declare.

\section{References}

1 G. Redlarski, B. Lewczuk, A. Żak, A. Koncicki, M. Krawczuk, J. Piechocki, K. Jakubiuk, P. Tojza, J. Jaworski, D. Ambroziak, Ł. Skarbek and D. Gradolewski, BioMed Res. Int., 2015, 2015, 1-18.

2 D. Soghomonyan, K. Trchounian and A. Trchounian, Appl. Microbiol. Biotechnol., 2016, 100, 4761-4771.

3 C. Hu, Z. Mou, G. Lu, N. Chen, Z. Dong, M. Hu and L. Qu, Phys. Chem. Chem. Phys., 2013, 15, 13038.

4 F. Qin and C. Brosseau, J. Appl. Phys., 2012, 111, 61301.

5 X. Zhao, Z. Zhang, L. Wang, K. Xi, Q. Cao, D. Wang, Y. Yang and Y. Du, Sci. Rep., 2013, 3, 3421.

6 G. Sun, B. Dong, M. Cao, B. Wei and C. Hu, Chem. Mater., 2011, 23, 1587-1593.

7 X.-J. Zhang, G.-S. Wang, W.-Q. Cao, Y.-Z. Wei, J.-F. Liang, L. Guo and M.-S. Cao, ACS Appl. Mater. Interfaces, 2014, 6, 7471-7478.

8 G. Li, T. Xie, S. Yang, J. Jin and J. Jiang, J. Phys. Chem. C, 2012, 116, 9196-9201.

9 X. F. Zhang, X. L. Dong, H. Huang, B. Lv, J. P. Lei and C. J. Choi, J. Phys. D: Appl. Phys., 2007, 40, 5383-5387.

10 B. Wen, M. Cao, M. Lu, W. Cao, H. Shi, J. Liu, X. Wang, H. Jin, X. Fang, W. Wang and J. Yuan, Adv. Mater., 2014, 26, 3484-3489.

11 P. Meng, K. Xiong, K. Ju, S. Li and G. Xu, J. Magn. Magn. Mater., 2015, 385, 407-411.
12 Y.-X. Gong, L. Zhen, J.-T. Jiang, C.-Y. Xu, W.-S. Wang and W.-Z. Shao, CrystEngComm, 2011, 13, 6839.

13 R. C. Che, L.-M. Peng, X. F. Duan, Q. Chen and X. L. Liang, Adv. Mater., 2004, 16, 401-405.

14 Z. Wang and G.-L. Zhao, Open J. Compos. Mater., 2013, 3, 1723.

15 Y. P. Duan, L. Wang, Z. Liu and S. C. Gu, Plast., Rubber Compos., 2013, 42, 82-87.

16 G. Wang, Z. Gao, S. Tang, C. Chen, F. Duan, S. Zhao, S. Lin, Y. Feng, L. Zhou and Y. Qin, ACS Nano, 2012, 6, 1100911017.

17 O. A. Al-Hartomy, A. A. Al-Ghamdi, F. Al-Salamy, N. Dishovsky, R. Shtarkova, V. Iliev and F. El-Tantawy, Int. J. Mater. Chem., 2012, 2, 116-122.

18 Y. Du, T. Liu, B. Yu, H. Gao, P. Xu, J. Wang, X. Wang and X. Han, Mater. Chem. Phys., 2012, 135, 884-891.

19 H. Xu, X. Yin, M. Zhu, M. Han, Z. Hou, X. Li, L. Zhang and L. Cheng, ACS Appl. Mater. Interfaces, 2017, 9, 6332-6341.

20 F. Moglie, D. Micheli, S. Laurenzi, M. Marchetti and V. Mariani Primiani, Carbon, 2012, 50, 1972-1980.

21 Z. Fang, C. Li, J. Sun, H. Zhang and J. Zhang, Carbon, 2007, 45, 2873-2879.

22 Y. Huang, Y. Wang, Z. Li, Z. Yang, C. Shen and C. He, J. Phys. Chem. C, 2014, 118, 26027-26032.

23 Y. Du, T. Liu, B. Yu, H. Gao, P. Xu, J. Wang, X. Wang and X. Han, Mater. Chem. Phys., 2012, 135, 884-891.

24 G. Li, T. Xie, S. Yang, J. Jin and J. Jiang, J. Phys. Chem. C, 2012, 116, 9196-9201.

25 J. Fricke and R. Petricevic, in Handbook of Porous Solids, Wiley-VCH Verlag GmbH, Weinheim, Germany, 2008, pp. 2037-2062.

26 M. A. Aegerter, N. Leventis and M. M. Koebel, Advances in SolGel Derived Materials and Technologies, 2011.

27 M. Koebel, A. Rigacci and P. Achard, J. Sol-Gel Sci. Technol., 2012, 63, 315-339.

28 A. M. ElKhatat and S. A. Al-Muhtaseb, Adv. Mater., 2011, 23, 2887-2903.

29 S. Chandrasekaran, P. G. Campbell, T. F. Baumann and M. A. Worsley, J. Mater. Res., 2017, 32, 4166-4185.

30 A. Borenstein, O. Hanna, R. Attias, S. Luski, T. Brousse and D. Aurbach, J. Mater. Chem. A, 2017, 5, 12653-12672.

31 N. Rey-Raap, A. Arenillas and J. A. Menéndez, in Submicron Porous Materials, Springer International Publishing, Cham, 2017, pp. 25-52.

32 M. J. Crane, M. B. Lim, X. Zhou and P. J. Pauzauskie, Microsyst. Nanoeng., 2017, 3, 17032.

33 A. Abolghasemi Mahani, S. Motahari and A. Mohebbi, Mar. Pollut. Bull., DOI: 10.1016/j.marpolbul.2017.10.012.

34 A. Allahbakhsh and A. R. Bahramian, RSC Adv., 2016, 6, 72777-72790.

35 X. X. C. Zhou and S. Geng, Carbon, 2016, 108, 234-241.

36 B. Liu, J. Li, L. Wang, J. Ren and Y. Xu, Composites, Part A, 2017, 97, 141-150.

37 Y. Du, W. Liu, R. Qiang, Y. Wang, X. Han, J. Ma and P. Xu, ACS Appl. Mater. Interfaces, 2014, 6, 12997-13006.

38 C. Song, X. Yin, M. Han, X. Li, Z. Hou, L. Zhang and L. Cheng, Carbon, 2017, 116, 50-58. 
39 M. Kruk and M. Jaroniec, Chem. Mater., 2001, 13, 3169-3183. 40 M. Thommes, K. Kaneko, A. V Neimark, J. P. Olivier, F. Rodriguez-Reinoso, J. Rouquerol and K. S. W. Sing, Pure Appl. Chem., 2015, 87, 1051-1069.

41 M. Thommes, Chem.-Ing.-Tech., 2010, 82, 1059-1073.

42 D. D. Do, E. A. Ustinov and H. D. Do, in Adsorption by Carbons, 2008, pp. 239-271.

43 M. A. Elsayed, P. J. Hall and M. J. Heslop, in Adsorption, 2007, vol. 13, pp. 299-306.

44 Y. Wang, B. Chang, D. Guan and X. Dong, J. Solid State Electrochem., 2015, 19, 1783-1791.

45 A. Kobayashi and G. I. Konishi, Molecules, 2009, 14, 364-377.

46 B. Chang, D. Guan, Y. Tian, Z. Yang and X. Dong, J. Hazard. Mater., 2013, 262, 256-264.

47 S. Motahari, B. S. Heidari and G. H. Motlagh, J. Appl. Polym. Sci., 2015, 132(37), 42543.

48 T. Zhang, B. Xiao, P. Zhou, L. Xia, G. Wen and H. Zhang, Nanotechnology, 2017, 28, 35.

49 W. Xie, X. Zhu, S. Yi, J. Kuang, H. Cheng, W. Tang and Y. Deng, Mater. Des., 2016, 90, 38-46.

50 L. Ding, A. Zhang, H. Lu, Y. Zhang and Y. Zheng, RSC Adv., 2015, 5, 83953-83959.

51 R. Kumar, A. P. Singh, M. Chand, R. P. Pant, R. K. Kotnala, S. K. Dhawan, R. B. Mathur and S. R. Dhakate, RSC Adv., 2014, 4, 23476.
52 X. Qiu, L. Wang, H. Zhu, Y. Guan and Q. Zhang, Nanoscale, 2017, 9, 7408-7418.

53 O. Levy and D. Stroud, Phys. Rev. B, 1997, 56, 8035-8046.

54 R. Qiang, Y. Du, Y. Wang, N. Wang, C. Tian, J. Ma, P. Xu and X. Han, Carbon, 2016, 98, 599-606.

55 L. Wang, X. Jia, Y. Li, F. Yang, L. Zhang, L. Liu, X. Ren and H. Yang, J. Mater. Chem. A, 2014, 2, 14940.

56 K. Sun, P. Xie, Z. Wang, et al., Polymer, 2017, 125, 50-57.

57 H. Bin Zhang, W. G. Zheng, Q. Yan, Z. G. Jiang and Z. Z. Yu, Carbon, 2012, 50, 5117-5125.

58 T. Wu, Y. Zhao, Y. Li, W. Wu and G. Tong, ChemCatChem, 2017, 9, 3486-3496.

59 Z. Ma, C.-T. Cao, Q.-F. Liu and J.-B. Wang, Chin. Phys. Lett., 2012, 29, 38401.

60 J. Jiang, D. Li, D. Geng, J. An, J. He, W. Liu and Z. Zhang, Nanoscale, 2014, 6, 3967-3971.

61 F. Meng, W. Wei, X. Chen, X. Xu, M. Jiang, L. Jun, Y. Wang and Z. Zhou, Phys. Chem. Chem. Phys., 2015, 18, 25102516.

62 F. Wen, H. Hou, J. Xiang, X. Zhang, Z. Su, S. Yuan and Z. Liu, Carbon, 2015, 89, 372-377.

63 M. Gholampoor, F. Movassagh-Alanagh and H. Salimkhani, Solid State Sci., 2017, 64, 51-61. 